\title{
Pitch and Loudness Tinnitus in Individuals with Presbycusis
}

\author{
Bruna Macangnin Seimetz ${ }^{1}$ Adriane Ribeiro Teixeira ${ }^{2}$ Leticia Petersen Schmidt Rosito ${ }^{3}$ \\ Leticia Sousa Flores ${ }^{4}$ Carlos Henrique Pappen ${ }^{5}$ Celso Dall'igna ${ }^{3}$
}

\footnotetext{
1 Program in Child and Adolescent Health, Universidade Federal do Rio Grande do Sul, Porto Alegre, Rio Grande do Sul, Brazil

2 Department of Developmental Psychology and Personality, Universidade Federal do Rio Grande do Sul, Instituto de Psicologia da UFRGS, Porto Alegre, Rio Grande do Sul, Brazil

${ }^{3}$ Department of Otolaryngology - Head and Neck Surgery, Hospital de Clínicas de Porto Alegre, Porto Alegre, Rio Grande do Sul, Brazil

${ }^{4}$ Audiologist Clinic, private office, Porto Alegre, Rio Grande do Sul, Brazil

${ }^{5}$ Medical School, Universidade Federal do Rio Grande do Sul, Porto Alegre, Rio Grande do Sul, Brazil
}

Address for correspondence Bruna Macangnin Seimetz, Program in Child and Adolescent Health, Universidade Federal do Rio Grande do Sul, Ramiro Barcelos, 2400 Porto Alegre, Rio Grande do Sul 90035-003, Brazil (e-mail: bruna.seimetz@gmail.com).

\begin{abstract}
Keywords

- presbycusis

- tinnitus

- aging

Introduction Tinnitus is a symptom that is often associated with presbycusis.

Objective This study aims to analyze the existence of association among hearing thresholds, pitch, and loudness of tinnitus in individuals with presbycusis, considering the gender variable.

Methods Cross-sectional, descriptive, and prospective study, whose sample consisted of individuals with tinnitus and diagnosis of presbycusis. For the evaluation, we performed anamnesis along with otoscopy, pure tone audiometry, and acuphenometry to analyze the psychoacoustic characteristics of tinnitus individuals.

Results The sample consisted of 49 subjects, with a mean age of $69.57 \pm 6.53$ years, who presented unilateral and bilateral tinnitus, therefore, a sample of 80 ears. In analyzing the results, as for acuphenometry, the loudness of tinnitus was more present at $0 \mathrm{~dB}$ and the pitch was $6 \mathrm{HKz}$ and $8 \mathrm{HKz}$. Regarding the analysis of the association between the frequency of greater hearing threshold and tinnitus pitch, no statistical significance ( $p=0.862)$ was found. As for the association between the intensity of greater hearing threshold and tinnitus loudness, no statistical significance $(p=0.115)$ was found.

Conclusion There is no significant association between the hearing loss of patients with presbycusis and the pitch and loudness of tinnitus.
\end{abstract}

\section{Introduction}

Presbycusis is a word derived from the Greek (presbus = elder + ákousis = hearing), which means reduced hearing acuity caused by aging. ${ }^{1}$ Its etiology is multifactorial and is characterized as bilateral, symmetrical, with slow and progressive evolution, mainly affecting high frequencies. It starts usually between 20 and 30 years of age, but becomes socially awkward from 40 to 50 years. $^{2}$ Studies show that there is an increase in the prevalence of presbycusis according to age, with a greater loss in high frequencies (from 80-89) and flattening out in individuals aged over 90 years. ${ }^{3,4}$ Presbycusis is a problem associated with senescence, with received

July 26, 2015

accepted

October 4, 2015

published online

February 1, 2016
DOI http://dx.doi.org/

10.1055/s-0035-1570311. ISSN 1809-9777.
Copyright $(2016$ by Thieme Publicações License terms Ltda, Rio de Janeiro, Brazil 
consequences to social and functional activities as well as to the psychological well-being of individuals. ${ }^{3,5,6}$

Tinnitus is a symptom related to the hearing system, which may be observed in individuals diagnosed with presbycusis. ${ }^{7}$ This is the conscious perception of sound that originates in the ears or head of the patient without the presence of an external source of sound generator, ${ }^{8}$ that is, the sensation of sound in the absence of an external sound stimulus. ${ }^{9}$ Its main origin is in the cochlea, and may be associated with various diseases that may spread to other auditory pathways. ${ }^{7}$

Ear diseases are the leading causes of tinnitus, but diseases that affect the ear secondarily, such as metabolic, cardiovascular, neurological, psychiatric, dental changes and possibly the consumption of drugs, caffeine, nicotine and alcohol may cause etiology. ${ }^{8}$

With respect to age, this is a symptom observed in adults and children, ${ }^{10}$ but its incidence increases with increasing age and also with hearing loss, making it a more common complaint in older individuals 7,10 and more common in men ${ }^{11}$ than women. It may be unilateral or bilateral, but in some cases patients can't refer to the side that sense, referring to the perception of sound inside the head, or, optionally, on the outside of the head. ${ }^{7,10}$

Psychoacoustic characteristics of tinnitus, which are frequency (pitch) and intensity (loudness), differ among patients, as well as in location and duration of tinnitus. ${ }^{8}$ The manner in which this symptom disturbs people's quality of life also varies from patient-to-patient, as they present different reactions. ${ }^{12}$ If gender is considered, literature data are controversial, since research shows that gender has no influence on the nuisance caused by tinnitus, ${ }^{13-15}$ reporting similarities in how men and women feel the symptom. However, there are studies that demonstrate greater nuisance in males, ${ }^{16}$ while others have reported greater discomfort among women. ${ }^{17,18}$ A recent study using functional imaging in patients with tinnitus reported differences in the activity of the orbitofrontal cortex extending to the frontopolar cortex of men and women with this symptom. ${ }^{15}$ The orbitofrontal cortex is an important tool for the emotional processing of sounds, justifying possible gender differences in the perception of tinnitus. ${ }^{15}$

Previous research have shown a relationship between tinnitus and hearing, which is more common and severe when associated with hearing loss. ${ }^{11,19}$ Another study also reported this relationship, with influence of frequency band and hearing thresholds. ${ }^{20}$

As an auditory perception or, in other words, a subjective characteristic, the conditions for research on tinnitus are limited. ${ }^{21}$ Nonetheless, given that this is a symptom with great impact in the lives of individuals, all scientific contribution in this area becomes important and necessary. 8,10

The assumptions described and the scarce national and international literature on research correlating hearing loss and the results found in the psychoacoustic measurements of tinnitus (pitch and loudness) justify the importance of this research.

The objective of this study is to analyze the existence of an association between hearing thresholds, pitch, and loudness of tinnitus in individuals with presbycusis, considering the gender variable.

\section{Methods}

This is a transversal, descriptive, and prospective study, whose sample consisted of individuals with presbycusis and tinnitus in the Chronic Tinnitus Clinic of the hospital of the institution that hosted this study.

The sample included individuals with tinnitus, whether unilateral or bilateral, diagnosed with presbycusis, and who underwent complete otorhinolaryngologic and audiological evaluation, with audiological exams (otoscopy, pure tone audiometry by air and bone conduction, acoustics, and acuphenometry impedance measures). The study excluded patients with a history of cognitive and / or neurological impairment observed during records analysis.

Initially, all patients were treated by an otorhinolaryngologist for the investigation of otologic and health history, as well as otoscopy.

Afterwards, all patients underwent an audiological evaluation conducted with pure tone audiometry performed in a soundproof booth, with audiometer Siemens, Unity PC model with HDA 200 headphones and bone vibrator B71 for research of the thresholds by air in the frequencies of $250 \mathrm{~Hz}, 500 \mathrm{~Hz}$, $1000 \mathrm{~Hz}, 2000 \mathrm{~Hz}, 3000 \mathrm{~Hz}, 4000 \mathrm{~Hz}, 6000 \mathrm{~Hz}$, and $8000 \mathrm{~Hz}$, and also bone conduction thresholds in the frequencies of $500 \mathrm{~Hz}$, $1000 \mathrm{~Hz}, 2000 \mathrm{~Hz}, 3000 \mathrm{~Hz}$, and $4000 \mathrm{~Hz}$.

Subsequently, we performed acuphenometry to analyze the psychoacoustic characteristics of each individual tinnitus, or we observed the sensation of pitch and loudness of the tinnitus. We found that pitch corresponds to the frequency of the symptom (low, medium, or high) and loudness to the intensity of it, which is equivalent to the volume of feeling reported by the pure tone of the patient or narrowband noise. ${ }^{8}$

The acuphenometry was performed using the procedures described by Branco-Barreiro, ${ }^{22}$ which initially was made to search for pitch. In all frequencies, we selected the ear of the individual threshold, added another $10 \mathrm{dBHL}$ and presented pure tone or noise, according to the patients' description on the characteristics of their tinnitus. We requested that the patient raise his or her hand when realizing that the sound presented was similar to his or her tinnitus. Next, we researched loudness. In the frequency indicated by the patient as similar to their tinnitus, the stimulus was presented (pure tone or noise), with initial intensity $10 \mathrm{dBHL}$ below the patient's threshold. Next, we increased intensity in steps of $2 \mathrm{dBHL}$, and the patients would raise their hand as soon as they realized that the intensity was similar to the presented tinnitus. This intensity was recorded and subtracted from the individual hearing threshold. This calculation enables the determination level of sensation. After the audiological evaluation, all subjects returned for consultation with the otorhinolaryngologist for the causal diagnosis of hearing loss and tinnitus.

The research had the approval of the institution's Ethics Committee, recorded under number 06026, as well as Informed Consent (IC) of all participating individuals. 
For data analysis, the sample was divided into subgroups according to the variable of interest, which were gender, frequency of greater hearing threshold, intensity of greater hearing threshold, pitch, and loudness obtained in acuphenometry. Regarding the frequency of greater hearing threshold and the pitch in acuphenometry, we analyzed the frequency bands analysis according to the following considerations: severe $(250 \mathrm{~Hz}$ and $500 \mathrm{~Hz})$, medium $(1000 \mathrm{~Hz}, 2000 \mathrm{~Hz}$, and $3000 \mathrm{~Hz})$, and acute $(4000 \mathrm{~Hz}, 6000 \mathrm{~Hz}$, and $8000 \mathrm{~Hz})$.

Regarding the intensity of greater hearing threshold, subgroups were divided according to individual threshold between 0 and $25 \mathrm{~dB}$ HL, 26 and $40 \mathrm{~dB}$ HL, 41 and $70 \mathrm{~dB} \mathrm{HL}, 71$ and $90 \mathrm{~dB} \mathrm{HL}$, and above $90 \mathrm{~dB}$ HL. As for loudness in acuphenometry, the group was divided into individuals who had tinnitus between 0 and $4 \mathrm{dBSL}, 5$ to $9 \mathrm{dBSL}, 10$ to $14 \mathrm{dBSL}, 15$ to $19 \mathrm{dBSL}, 20$ to $24 \mathrm{dBSL}, 25$ to $30 \mathrm{dBSL}$, and above 30 dBSL.

We entered the data into a database built in Microsoft Excel. We performed the analysis using SPSS software version 18 , with the correlation analysis through the Pearson correlation coefficient, Student's $t$-test for comparison of independent means, and Fisher's Exact Test. We considered a significance level of $5 \%(p<0.05)$.

\section{Results}

The study sample consisted of 49 subjects, with a mean age of $69.57 \pm 6.53$ years, minimum age of 53 years and maximum of 85 years. As for gender, the study included 28 female subjects (57.15\%) and 21 male (42.85\%). Of these, 7 (14.28\%) had tinnitus in the right ear (RE), 11 (22.44\%) in the left ear (LE), and 31 (63.26\%) in both ears (BE). Thus, for data analysis, we considered only the ears that had tinnitus, totalizing 80 ears, $38 \mathrm{RE}$ and $42 \mathrm{LE}$.

- Table 1 shows the frequency distribution of the individuals according to the intensity of greater hearing threshold. In analyzing the results, we observed that most of the patients evaluated had their greatest auditory thresholds between 50 and $75 \mathrm{~dB}$ HL. The average loudness of tinnitus was $17.9 \mathrm{~dB}$ HL. - Table 2 shows the frequency distribution of the loudness of tinnitus obtained in acuphenometry. Most of the evaluated ears (80.1\%) had tinnitus loudness between $0 \mathrm{~dB}$ HL and $19 \mathrm{~dB}$ HL.

- Table 3 shows the comparison between genders with regards to the characteristics of greater hearing threshold (frequency and intensity) and psychoacoustic characteristics of tinnitus (pitch and loudness). We concluded that there is no significant difference between genders comparing the average frequency of greater hearing threshold ( $p=0.889)$, intensity of greater hearing threshold $(p=0.140)$, pitch obtained in acuphenometry $(p=0.144)$, and loudness obtained in acuphenometry ( $p=0.139)$.

- Table 4 shows the analysis between gender and two other variables, loudness and frequency range, obtained in acuphenometry. There was no significant association in both comparisons, with $p=0.603$ and $p=0.555$, respectively.

In - Table 5, we present the frequency distribution of patients according to frequency of greater hearing threshold
Table 1 Distribution of frequency of ears evaluated according to the intensity of greater hearing threshold

\begin{tabular}{|l|l|l|}
\hline $\begin{array}{l}\text { Intensity of greater hearing } \\
\text { threshold (dB HL) }\end{array}$ & $\mathrm{N}$ & $\%$ \\
\hline $30|-| 35$ & 7 & 8.8 \\
\hline 40 | - 45 & 10 & 12.6 \\
\hline $50|-| 55$ & 17 & 21.3 \\
\hline $60|-| 65$ & 20 & 25.1 \\
\hline $70|-| 75$ & 14 & 17.5 \\
\hline $80|-| 85$ & 6 & 7.5 \\
\hline $90|-| 95$ & 4 & 5.1 \\
\hline 110 & 2 & 2.5 \\
\hline Total & 80 & 100.0 \\
\hline
\end{tabular}

Abbreviations: $\mathrm{dB} \mathrm{HL}$, decibel hearing level; N, number; \%, percentage.

Table 2 Distribution of frequency of ears evaluated according to the loudness of tinnitus obtained in acuphenometry

\begin{tabular}{|l|l|l|}
\hline Acuphenometry - Loudness (dBSL) & N & $\%$ \\
\hline $0|-| 4$ & 19 & 23.7 \\
\hline $5|-| 9$ & 17 & 21.3 \\
\hline $10|-| 14$ & 12 & 15.1 \\
\hline $15|-| 19$ & 16 & 20.0 \\
\hline $20|-| 24$ & 7 & 8.8 \\
\hline $25|-| 30$ & 6 & 7.6 \\
\hline$<30$ & 3 & 3.8 \\
\hline Total & 80 & 100.0 \\
\hline
\end{tabular}

Abbreviation: (dBSL), decibel sensation level; $\mathrm{N}$, number; \%, percentage.

and pitch in acuphenometry. We found low correlation $(r=0.080)$ and no significance $(p=0.478)$.

Regarding the analysis of the frequency range of greater hearing threshold (divided into severe, medium, and high), and pitch obtained in acuphenometry (ranked in the same way), we observed no significant association $(p=0.081)$ (-Table 6).

- Table 7 demonstrates the analysis of the intensity ratings of greater hearing threshold and loudness in acuphenometry, whereby it is possible to conclude that there was no significant association between these variables $(p=0.115)$.

\section{Discussion}

The results of this study show that the mean age observed in the sample (69.57 \pm 6.53 years) was consistent with other studies investigating tinnitus in the elderly. Previous research found average ages of 69.53 years $^{23}$ and 65.5 years. $^{24}$

As for tinnitus location, some studies corroborate this research, primarily finding bilateral tinnitus, followed by unilateral LE, and, finally, unilateral RE. ${ }^{18,25,27}$ 
Table 3 Comparison of the gender characteristics of greater hearing threshold (frequency and intensity) and psychoacoustic characteristics of tinnitus (pitch and loudness)

\begin{tabular}{|l|l|l|l|l|l|}
\hline Variables & Gender & N & Average & Standard deviation & p-value \\
\hline Often the higher threshold & F & 48 & 6791.7 & 1878.9 & 0.889 \\
\hline & M & 32 & 6851.6 & 1856.6 & \\
\hline Higher threshold intensity & F & 48 & 58.5 & 17.2 & 0.140 \\
\hline & M & 32 & 64.4 & 17.1 & \\
\hline Pitch (acuphenometry) & F & 48 & 3869.8 & 2597.0 & 0.144 \\
\hline & M & 32 & 4781.3 & 2867.2 & \\
\hline Loudness (acuphenometry) & F & 48 & 12.3 & 10.7 & 0.139 \\
\hline & M & 32 & 9.1 & 7.2 & \\
\hline
\end{tabular}

Abbreviations: $F$, female; $M$, male; $N$, number.

Student $t$-test, with significance level of $5 \%(p<0.05)$.

Table 4 Analysis of the influence of gender on the acuphenometry loudness and frequency range obtained in acuphenometry

\begin{tabular}{|c|c|c|c|c|c|c|c|c|c|c|c|c|}
\hline \multirow{3}{*}{ Gender } & \multicolumn{12}{|c|}{ Acuphenometry } \\
\hline & \multicolumn{7}{|c|}{ Loudness (dB HL) } & \multirow[t]{2}{*}{$\mathrm{p}$-value } & \multicolumn{4}{|c|}{ Frequency Range } \\
\hline & $\begin{array}{ccc}0 & 1 \\
-1 & 4\end{array}$ & $\begin{array}{lll}5 & 1 \\
-1 & 9\end{array}$ & $\begin{array}{l}10 \mid \\
-\mid 14\end{array}$ & $\begin{array}{lll}15 & \mid \\
-I & 19\end{array}$ & $\begin{array}{l}201 \\
-124\end{array}$ & $\begin{array}{l}251 \\
-130\end{array}$ & $<30$ & & Serious & Medium & Acute & p-value \\
\hline $\begin{array}{l}\mathrm{F} \\
(\mathrm{n}=48)\end{array}$ & 11 & 8 & 8 & 9 & 4 & 4 & 4 & 0.603 & 8 & 14 & 26 & 0.555 \\
\hline $\begin{array}{l}M \\
(n=32)\end{array}$ & 8 & 9 & 4 & 7 & 3 & 1 & 0 & & 3 & 8 & 21 & \\
\hline Total & 19 & 17 & 12 & 16 & 7 & 5 & 4 & & 11 & 22 & 47 & \\
\hline
\end{tabular}

Abbreviations: $\mathrm{dB} \mathrm{HL}$, decibel hearing level: $\mathrm{F}$, female; $\mathrm{M}$, male.

Fisher's exact test with a significance level of $5 \%(p<0.05)$.

Table 5 Frequency analysis of greater hearing threshold and the pitch in acuphenometry

\begin{tabular}{|l|l|l|l|l|}
\hline & \multicolumn{2}{|l|}{$\begin{array}{l}\text { Greater frequency } \\
\text { hearing threshold }\end{array}$} & \multicolumn{2}{l|}{$\begin{array}{l}\text { Pitch in } \\
\text { Acuphenometry }\end{array}$} \\
\hline Frequency & $\mathrm{N}$ & $\%$ & $\mathrm{~N}$ & $\%$ \\
\hline 250 & 1 & 1.3 & 3 & 3.8 \\
\hline 500 & - & - & 8 & 10.0 \\
\hline 1000 & 1 & 1.3 & 7 & 8.8 \\
\hline 2000 & 1 & 1.3 & 10 & 12.5 \\
\hline 3000 & 4 & 5.0 & 5 & 6.3 \\
\hline 4000 & 4 & 5.0 & 12 & 15.0 \\
\hline 6000 & 19 & 23.8 & 18 & 22.5 \\
\hline 8000 & 50 & 62.5 & 17 & 21.3 \\
\hline Total & 80 & 100.0 & 80 & 100.0 \\
\hline
\end{tabular}

Abbreviations: $\mathrm{N}$, number; \%, percentage.

Pearson's correlation coefficient $(r)$, with a significance level of $5 \%$

$(p<0.05)$.

$r=0.80$.

$p=0.478$.

Considering gender, we observed that the number of females (57.15\%) was higher than men. Previous studies confirm our results, varying between $51 \%$ and $64 \%$ women in their samples. ${ }^{18,25-29}$ A possible explanation to be considered is the fact that women are more careful and concerned about their health, seeking further medical care and, thus, show higher prevalence in studies and research. ${ }^{12}$ Moreover, given that the sample of this study consisted of elderly people, we must mention the higher proportion of senior females in Brazil as a possible factor in female prevalence. ${ }^{30}$ Research shows that in 2000 , for every 100 elderly women there were 81 elderly men. By 2050, this ratio will be 100 elderly women to 76 elderly men. ${ }^{31}$

We did not find previous literary data to corroborate the findings of this study in terms of psychoacoustic characteristics of tinnitus in patients with presbycusis, comparing gender and pitch and loudness of tinnitus (-Tables 3 and 4). However, a study conducted with 607 women and 573 men with chronic tinnitus with an average age of 50 years observed an average pitch in women of $5.7 \mathrm{~Hz}$ and $5.4 \mathrm{~Hz}$ for right and left ears, respectively. ${ }^{18}$ As for men, average pitch was $5.7 \mathrm{~Hz}$ for both ears. ${ }^{18}$ This is gender indifference is in accordance with the present study. More recent research, however, differs from our data, finding higher pitch value in women (average 3963.2 and $3602 \mathrm{~Hz}$ for right and left ears, respectively) than in men (3047.6 in RE and $3228 \mathrm{~Hz}$ in LE). ${ }^{32}$ Regarding the loudness of tinnitus, we observed differences between this study and previous studies, ${ }^{16,18}$ where the authors report significantly higher loudness in men 
Table 6 Analysis of the frequency range of greater hearing threshold and pitch obtained in acuphenometry, by frequency bands

\begin{tabular}{|l|l|l|l|l|l|}
\hline \multirow{2}{*}{ Frequency range of greater hearing threshold } & Acuphenometry - Frequency Range & & \\
\cline { 2 - 7 } & Serious & Average & Acute & Total & p-value $^{*}$ \\
\hline Serious & - & - & 1 & 1 & 0.081 \\
\hline Average & 1 & 4 & 1 & 6 & \\
\hline Acute & 10 & 18 & 45 & 73 & \\
\hline Total & 11 & 22 & 47 & 80 & \\
\hline
\end{tabular}

Fisher's exact test with a significance level of $5 \%(p<0.05)$.

Table 7 Analysis of the intensity ratings of greater hearing threshold and the loudness in acuphenometry

\begin{tabular}{|c|c|c|c|c|c|c|c|c|c|}
\hline \multirow[t]{2}{*}{ Greater threshold intensity } & \multicolumn{7}{|c|}{ Acuphenometry - Loudness (dB HL) } & \multirow[t]{2}{*}{ Total } & \multirow[b]{2}{*}{ p-value } \\
\hline & 0 I-I 4 & $5|-| 9$ & 10 I-I 14 & 15 I-I 19 & 20 I-I 24 & 25 I-I 30 & $<30$ & & \\
\hline $26|-| 40$ & 1 & 3 & - & 5 & 2 & 1 & - & 12 & \multirow[t]{4}{*}{0.115} \\
\hline $41|-| 70$ & 14 & 9 & 12 & 6 & 5 & 3 & 3 & 52 & \\
\hline $71|-| 90$ & 3 & 5 & - & 3 & - & 1 & 1 & 13 & \\
\hline$<90$ & 1 & - & - & 2 & - & - & - & 3 & \\
\hline Total & 19 & 17 & 12 & 16 & 7 & 5 & 4 & 80 & \\
\hline
\end{tabular}

Abbreviation: $\mathrm{dB} \mathrm{HL}$, decibel hearing level.

Fisher's exact test with a significance level of $5 \%(p<0.05)$.

(42.3 $\mathrm{dB}$ for $\mathrm{RE}$ and $44.3 \mathrm{~dB}$ for $\mathrm{LE}$ ) compared to women (35.7 dB for RE and $37.3 \mathrm{~dB}$ for $\mathrm{LE}){ }^{18}$

When analyzing frequency of greater hearing threshold ( $\mathbf{- T a b l e s} 5$ and $\mathbf{6}$ ), we observed higher thresholds at high frequencies. This is in agreement with the audiological profile that characterizes presbycusis, a sensorineural hearing loss with downward audiometric curve. other studies in elderly patients with tinnitus also reported this audiological pattern. ${ }^{3,5,23}$ Another study performed in the state of Rio Grande do Sul in Brazil involving a sample of 215 elderly also showed that the frequencies with greater hearing thresholds were $6000 \mathrm{~Hz}$ and $8000 \mathrm{~Hz}^{33}$

Regarding tinnitus pitch, this study found that most of the evaluated ears had a high pitch, which corroborates previous research. ${ }^{26,32,34,35}$ Despite the fact that frequency of greater hearing threshold and pitch in acuphenometry are located in the range of high frequencies, corroborating the study of Noreña et al., ${ }^{36}$ the correlation between these variables is weak. Other studies also report a poor correlation between the pitch of the tinnitus and hearing loss frequencies, or even no correlation, ${ }^{25,29,37-40}$ although number of ears examined may have an influence in this trend. In fact, some authors found a positive correlation between tinnitus pitch and frequency with greater hearing loss. ${ }^{38}$

The greater the intensity of the auditory threshold was found that in most of the individuals ranged between 40 and 65dBNA, which is also compatible with presbycusis above and is comparable with previous studies. ${ }^{33}$ In relation to the loudness of the tinnitus, it was found that the majority of cases varied between 0 and $19 \mathrm{dBSL}$, corroborated by a recent study where only $8 \%$ of the sample had tinnitus loudness equal to or greater than $20 \mathrm{dBSL}^{32}$ The average of this feature
(17.9 dBSL) is higher than the value reported in previous research, where the average was $14.1 \mathrm{~dB} .{ }^{41}$ However, the difference between studies may be due to sample average age (32.1 years) was younger than the sample of the present research.

The analysis of the intensity of greater hearing threshold and the loudness of tinnitus showed no significant association. In the literature, were not found previous studies correlating these variables. However, this finding may have been due to the fact of acuphenometry be a subjective test that depends on the intellectual capacity and concentration of the patient at the time of performing the test, ${ }^{42}$ and thus can restrict the results. ${ }^{18}$

\section{Conclusions}

This study showed no association between hearing loss, gender, pitch, and loudness of tinnitus in individuals with presbycusis. However, the studied literature still has significantly controversial data on such characteristics, highlighting the importance of further research on the topic.

\section{References}

1 Priberam Dictionary of the Portuguese Language (2013). . Available at: http://www.priberam.pt/dlpo/presbiacusia. Accessed on Oct 11, 2013

2 Roth TN, Hanebuth D, Probst R. Prevalence of age-related hearing loss in Europe: a review. Eur Arch Otorhinolaryngol 2011;268(8): 1101-1107

3 Baraldi GS, de Almeida LC, Borges ACC. Hearing loss in aging. Braz J Otorhinolaryngol 2007;73(1):58-64 
4 Teixeira AR, Freitas CR, Millão LF, et al. Relationship Among Hearing Loss, Age, Gender and Senior Quality of Life. Int Arch Otorhinolaryngol 2008;12:62-70

5 Guerra TM, Estevanovic LP, Cavalcante MdeÁ, Silva RCL, Miranda ICC, Quintas VG. Profile of audiometric thresholds and tympanometric curve of elderly patients. Braz J Otorhinolaryngol 2010; 76(5):663-666

6 Ciorba A, Bianchini C, Pelucchi S, Pastore A. The impact of hearing loss on the quality of life of elderly adults. Clin Interv Aging 2012; 7:159-163

7 Assumption ARM, Albertino S. Tinnitus Rev. Pedro Ernesto Univ Hosp UERJ 2012;11:19-22

8 Sanchez TG, Ferrari GMS. What is tinnitus? In: Samelli AG. Tinnitus: assessment, diagnosis and rehabilitation. Current approaches. São Paulo: Lovise; 2004. p. 17-22.

9 Gonçalves MS, Tochetto TM, Rossi AG. Condição auditiva de indivíduos com queixa de zumbido. Saúde 2005;31:5-9

10 Baguley DM. Mechanisms of tinnitus. Br Med Bull 2002;63: 195-212

11 Axelsson A, Ringdahl A. Tinnitus-a study of its prevalence and characteristics. Br J Audiol 1989;23(1):53-62

12 Coelho CCB, Sanchez TG, Bento RF. Características do zumbido em pacientes atendidos em serviço de referência. Int Arch Otorhinolaryngol 2004;8:216-224

13 Méric C, Gartner M, Collet L, Chéry-Croze S. Psychopathological profile of tinnitus sufferers: evidence concerning the relationship between tinnitus features and impact on life. Audiol Neurootol 1998;3(4):240-252

14 Pinto PC, Sanchez TG, Tomita S. The impact of gender, age and hearing loss on tinnitus severity. Braz J Otorhinolaryngol 2010; 76(1):18-24

15 Vanneste S, Joos K, De Ridder D. Prefrontal cortex based sex differences in tinnitus perception: same tinnitus intensity, same tinnitus distress, different mood. PLoS ONE 2012;7(2):e31182

16 Hiller W, Goebel G. Factors influencing tinnitus loudness and annoyance. Arch Otolaryngol Head Neck Surg 2006;132(12): 1323-1330

17 Welch D, Dawes PJ. Personality and perception of tinnitus. Ear Hear 2008;29(5):684-692

18 Seydel C, Haupt H, Olze H, Szczepek AJ, Mazurek B. Gender and Chronic Tinnitus: differences in tinnitus-related distress depend on age and duration of tinnitus. Ear Hear 2013;34:661-672

19 Lindgren T, Wieslander G, Dammström BG, Norbäck D. Tinnitus among airline pilots: prevalence and effects of age, flight experience, and other noise. Aviat Space Environ Med 2009;80(2):112-116

20 Brown SC. Older Americans and Tinnitus: A Demographic Study and Chartbook [dissertation]. Washington, USA: Gallaudet University Gallaudet Research Institute; 1990:1-22

21 Sanchez TG, Zonato AI, Bittar RSM, Bento RF. Controvérsias sobre a fisiologia do zumbido. Int Arch Otorhinolaryngol 1997;1:2-8

22 White-Barreiro FCA. Basic Audiological Evaluation and Psychoacoustics of Tinnitus. In: Samelli AG. Tinnitus: assessment, diagnosis and rehabilitation. Current approaches. São Paulo: Lovise; 2004. p. 55-9.

23 Ferreira LMBM, Ramos Júnior AN, Mendes EP. Characterization of tinnitus in the elderly and its possible related disorders. Braz J Otorhinolaryngol 2009;75(2):249-255

24 Moura LOS, Iorio MCM, Azevedo MF. A eficácia da adaptação de prótese auditiva na redução ou eliminação do zumbido. Braz J Otorhinolaryngol 2004;70:624-631

25 Figueiredo RR, Rates MA, Azevedo AA, Oliveira PM, Navarro PBA Correlation analysis of hearing thresholds, validated questionnaires and psychoacoustic measurements in tinnitus patients. Braz J Otorhinolaryngol 2010;76(4):522-526
26 Urnau D, Tochetto TM. Characteristics of tinnitus and hyperacusis in normal hearing individuals. Int Arch Otorhinolaryngol 2011; $15: 468-474$

27 Mondelli MFCG, Rocha AB. Correlation between the audiologic findings and buzz disturbing. Int Arch Otorhinolaryngol 2011; $15: 172-180$

28 Lasisi AO, Abiona T, Gureje O. Tinnitus in the elderly: Profile, correlates, and impact in the Nigerian Study of Ageing. Otolaryngol Head Neck Surg 2010;143(4):510-515

29 Karatas E, Deniz M. The comparison of acoustic and psychic parameters of subjective tinnitus. Eur Arch Otorhinolaryngol 2012;269(2):441-447

30 Camarano AA. Aging of the population: a demographic contribution. In: EV Freitas, Py G, Neri AL, Cançado FAX, Gorzoni ML, SM Rocha. Treaty of Geriatrics and Gerontology. 2. ed. Rio de Janeiro: Guanabara Koogan; 2006:88-105

31 Carvalho JAM, Wong LR. The changing age structure of the population in the first half of the XXI century. Cad Saude Publica 2008;24:597-605

32 Makar SK, Biswas A, Shatapathy P. The impact of tinnitus on sufferers in Indian population. Indian J Otolaryngol Head Neck Surg 2014;66(Suppl 1):37-51

33 Seimetz BM, Flores LS, Copetti NS, Freitas NB, Gonçalves AK, Teixeira AR. Hearing thresholds of a group of elderly. Proceedings of the 28th International Meeting of Audiology; April 24-27, 2013; Salvador, Brazil. São Paulo, Brazilian Academy of Audiology; 2013. p. 82.

34 Menezes P, Santos Filha VAV. Acuphenometry: the rescue of an assessment instrument of tinnitus and its correlation with sensory hearing loss. Rev Fonoaudiol Bras 2005;3:1-4

35 Shekhawat GS, Searchfield GD, Stinear CM. The relationship between tinnitus pitch and hearing sensitivity. Eur Arch Otorhinolaryngol 2014;271(1):41-48

36 Noreña A, Micheyl C, Chéry-Croze S, Collet L. Psychoacoustic characterization of the tinnitus spectrum: implications for the underlying mechanisms of tinnitus. Audiol Neurootol 2002;7(6): 358-369

37 Sereda M, Hall DA, Bosnyák DJ, et al. Re-examining the relationship between audiometric profile and tinnitus pitch. Int J Audiol 2011; 50(5):303-312

38 Schecklmann M, Vielsmeier V, Steffens T, Landgrebe M, Langguth B, Kleinjung T. Relationship between Audiometric slope and tinnitus pitch in tinnitus patients: insights into the mechanisms of tinnitus generation. PLoS ONE 2012;7(4):e34878

39 Silva AL, Butzke BL, Deutsch KM, et al. Correlation between acuphenometry and audiometry in patients with chronic tinnitus. Proceedings of the 32nd Scientific Week of the Porto Alegre University Hospital; 2012 August; Porto Alegre, Brazil. Porto Alegre, Rev. HCPA \& Fac. Med. Univ. Fed. Rio Gd. South 2012.; 32 (Suppl.) 100.

40 Pan T, Tyler RS, Ji H, Coelho C, Gehringer AK, Gogel SA. The relationship between tinnitus pitch and the audiogram. Int J Audiol 2009;48(5):277-294

41 Schecklmann M, Vielsmeier V, Steffens T, Landgrebe M, Langguth B, Kleinjung T. Relationship between Audiometric slope and tinnitus pitch in tinnitus patients: insights into the mechanisms of tinnitus generation. PLoS ONE 2012;7(4):e34878

42 Morais AA, Gil D. Tinnitus in individuals without hearing loss and its relationship with temporomandibular dysfunction. Braz J Otorhinolaryngol 2012;78:59-65

43 Aparecida de Azevedo A, Mello de Oliveira P, Gomes de Siqueira A, Figueiredo RR. A critical analysis of tinnitus measuring methods. Braz J Otorhinolaryngol 2007;73(3):418-423 